\title{
Numerical simulations of the piston effect for near-critical fluids in spherical cells under small thermal disturbance
}

\author{
Zhan-Chao $\mathrm{Hu}^{\mathrm{a}}$, Xin-Rong Zhang ${ }^{\mathrm{a}, \mathrm{b}, *}$ \\ ${ }^{a}$ Department of Energy and Resources Engineering, College of Engineering, Peking University, \\ Beijing 100871, China \\ ${ }^{b}$ Beijing Engineering Research Center of City Heat, Peking University, Beijing 100871, China
}

\begin{abstract}
Near the liquid-vapor critical point, strong anomalies in thermodynamic and transport properties induce the piston effect. In the present study, the piston effect is investigated in spherical cells filled with near-critical fluids under zero gravity and small thermal disturbance conditions by numerically solving the thermodynamic model. The results show typical fast-responding bulk temperature, together with high and stable temperature efficiency, which is determined by the particular nature of near-critical fluids. Particularly, a nondimensionalization method is developed to study the influences of the specific heat ratio on the piston effect by simulations for eight near-critical fluids. Results show the specific heat ratio is an efficient indicator, which is negatively correlated with the nondimensional bulk temperature change. The influences of the characteristic length and the boundary conditions on the piston effect are also studied for near-critical carbon dioxide.
\end{abstract}

${ }^{*}$ Corresponding author at: Department of Energy and Resources Engineering, College of Engineering, Peking University, Beijing 100871, China. Tel.: +86 10 62755052; fax: +86 10 62755052 .

Email address: xrzhang@coe.pku.edu.cn (Xin-Rong Zhang) 
In addition, some rules concerning the boundary heat flux and the overall heat transfer are also discussed and concluded.

Keywords: Near-critical fluids; Piston effect; Numerical simulations.

\section{Introduction}

The liquid-vapor critical point is the end point of the pressure-temperature curve under which a liquid and its vapor can coexist. At the liquid-vapor critical point, defined by a critical pressure $P_{c}$ and a critical density $\rho_{c}$, phase boundaries vanish. A supercritical fluid is any substance at a temperature and pressure above its critical point. In this study, near-critical fluid refers to a supercritical fluid whose state slightly above critical state. Approaching the liquid-vapor critical point, strong anomalies can be found in thermal and transport coefficients. For example, both of the isothermal compressibility and thermal expansion grow dramatically while the thermal diffusivity tends to zero [1]. The peculiar thermophysical properties of fluids induce a new mode of heat transfer in fixed-volume cells filled with near-critical fluids, which is called the piston effect (PE) [2, 3, 4].

In microgravity environment, the thermal relaxation of a near-critical fluid was long thought to be infinitely slow without convection, which was termed as the critical slowing down. However, in 1985, Nitsche and Straub [5] carried out a space experiment and found the temperature at the center of the fluid following the rising wall temperature closely, which meant they actually observed a critical speeding up. Early theoretical studies mainly focused on the physical process of the PE. In 1990, three independent teams [2, 3, 4] put forward their explanations using different approaches. Afterwards, Zappoli and Carlès [6] applied matched asymptotic expansions and multiple-time-scale techniques to solve 
the one-dimensional compressible Navier-Stokes equations for a van der Waals gas and revealed the leading role of the acoustic waves in the PE. Then they [7] used matched asymptotic descriptions techniques and noted that ignoring the influence of bulk viscosity, energy should be transported by acoustic process very close to the critical point. And in this regime, the reflection rules of acoustic waves on thermostatted boundaries are inverted [8]. The amplitude of the reflected wave changes of sign, which is different from normal compressible gases. Moreover, Carlès [9] discovered the existence of viscous regime, during which going closer to the critical point, the heat transfer then slows down. As for density relaxation, Bailly and Zappoli [10] presented a complete hydrodynamic theory of density relaxation. They used matched asymptotic expansion technique to solve one-dimensional Navier Stokes equations and noted that the density relaxation is governed by the PE and heat diffusion. The whole process can be summarized as an expansion-compression zone traveling at the diffusion velocity and ultimately disappearing before reaching the other end of the sample cell. Early experimental studies $[11,12,13,14]$ mainly focused on the verification of the PE.

In 2006, Miura et al. [15] made the first experimental observation of the thermally induced acoustic waves. Then part of their experimental data was verified analytically by Carlès [16] using asymptotic methods. Onuki [17] presented a general theory of thermoacoustic waves involving the heat conduction in the boundary walls and bulk viscosity. Afterwards, the nature of thermoacoustic waves attracted increasing attention. Shen and Zhang studied the thermoacoustic waves emission, propagation and reflection under different temperature boundary conditions by solving the governing hydrodynamic equations [18]. Besides, they studied the thermoacoustic waves emission under internal and boundary thermal disturbance 
[19]. Hasan and Farouk also studied the thermoacoustic waves generation under different boundary heating rate using a real-fluid CFD model [20]. The results provided details regarding the thermal transport mechanisms at near-critical and pseudo-critical state fluids, and showed that the heating rate and the initial reduced temperature (i.e. $\varepsilon=\left(T_{0}-T_{c}\right) / T_{c}$, where $T_{0}$ and $T_{c}$ are initial and critical temperature) are key factors in the generation of acoustic waves.

The interplay between buoyant convection and PE has also been an active research topic. Zappoli et al. conducted a series of two-dimensional [21, 22, 23] and three-dimensional [24] numerical simulations for near-critical van der Waals fluid by a finite volume method together with an acoustic filtering procedure. A similar problem was further studied by Shen and Zhang [25, 26]. Notably, an unexpected temperature oscillations at the convection onset in terms of the PE were found by Amiroudine and Zappoli [27]. The Rayleigh-Taylor-like gravitational instability and thermovibrational instability in near-critical fluids were also studied by Amiroudine et al. [28, 29]. A monograph by Zappoli et al. [30] and a review by Shen and Zhang [31] give a detailed summary of previous researches on this topic.

In this paper, the PE in spherical cells filled with near-critical fluids under zero gravity condition is studied numerically to identify how some key factors influence the PE. The thermodynamic model presented by Boukari et al. [3] is used, and an explicit finite difference method using a central difference scheme for the spatial discretization is developed to solve the model iteratively. The NIST database [32] is used to obtain the $\rho-P-T$ relations for near-critical fluids.

The important factors we study in this paper are the specific heat ratio, the characteristic length and the boundary condition. As for the specific heat ratio, 
some previous studies $[33,34]$ have revealed the relationship between it and the bulk temperature increase. However, their conclusions are drawn from the PE for one near-critical fluid. In the present study, the PE for different near-critical fluids is further studied to show how the specific heat ratio influences the PE. In order to make different fluids be able to be compared with each other, a nondimensionalization method is particularly developed. Regarding the characteristic length, it is an important geometrical factor that changes the physical model. The PE under different characteristic lengths must differ greatly. With regard to the boundary condition, previous studies only focused on the influences of boundary condition on the thermoacoustic process. Thus, it is also significant to study the problem on a longer timescale and deepen our understandings from a macroscopic view. The last two factors are studied for near-critical $\mathrm{CO}_{2}$, so it is not necessary to nondimensionalize variables.

Besides, it should be mentioned here that small thermal disturbance is considered only in the present study, because under strong thermal disturbance, the initial reduced temperature (i.e. $\varepsilon$, which can be seen as the distance to critical point) will be altered during the PE. As a result, the nature of a near-critical fluid will change dramatically and the controlled variable is altered. For example, when we study the influences of the characteristic length on the PE, the strong thermal disturbance will change the specific heat ratio considerably too. As a result, it is difficult to distinguish the dominant factor.

In Section 2, we introduce the problem formulation and the numerical method used in simulations. An explicit finite difference method using a central difference scheme for the spatial discretization is relied on solving the governing equations, whose validity is also discussed. In Section 3, related definitions of physical quan- 
tities are presented. In Section 4, how the specific heat ratio, the characteristic length and the boundary condition, influence the PE is shown and discussed. The paper is finally concluded in Section 5.

\section{Physical and numerical model}

\subsection{Physical model}

As shown in Fig.1, the physical model is a spherical cell filled with nearcritical fluid under zero gravity condition. $R$ is the radius of the cell and the origin $O$ is placed at the surface of the sphere. Being highly symmetrical, the spherical cell was adapted by Straub et al. [12, 35, 36] in their space experiments, which certified the presence of the PE both in one-phase and two-phase region, and evaluated the isochoric heat capacity measurements at the critical isochore of $\mathrm{SF}_{6}$. On the other hand, the critical pressure for most fluids are much higher than standard atmospheric pressure. Owing to good pressure bearing ability, many pressure vessels are designed as spherical geometry. Thus a spherical cell filled with one near-critical fluid is a reasonable configuration in experiments and applications.

Initially, the fluid is in thermodynamic equilibrium. The density and temperature are uniform throughout the whole cell. The initial conditions are

$$
\begin{gathered}
\rho_{0}=\rho_{c}, \\
T_{0}=(1+\varepsilon) T_{c},
\end{gathered}
$$

with $T_{c}$ the critical temperature, $\rho_{c}$ the critical density, $\varepsilon$ the initial reduced temperature and $\varepsilon=\left(T_{0}-T_{c}\right) / T_{c}$. At $x=0$, the boundary conditions used in this study are of the Dirichlet type:

$$
T_{\text {wall }}=T_{0}+\Delta T_{\text {wall }},
$$




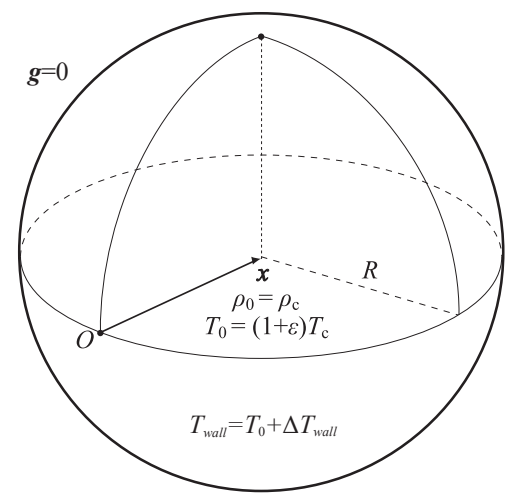

Figure 1: Physical model. $R$ is the radius of the cell and the origin $O$ is placed at the surface of the sphere.

namely that the boundary temperature suddenly rises by $\Delta T_{\text {wall }}$ at $t=0 \mathrm{~s}$. With clear physical meaning, this type of boundary condition is also used in some previous studies [33, 34, 37]. But it is not directly realizable in experiments $[12,14]$ under the influence of heat capacity of a container. In particular, Garrabos et al. [14] pointed out the relevance of applying identical total energy to make comparable simulations and experiments. A symmetry boundary condition is applied for $x=R$ in this study, which is equivalent to a Neumann boundary condition with the value of heat flux set to zero.

\subsection{Mathematical model and numerical method}

There are two main methods that can be used to simulate the PE. One is to solve the complete hydrodynamic description of an isotropic, Newtonian, compressible, and dissipative (viscous and heat-conducting) fluid. The equations were first solved by Zappoli et al. [4] and the essence of the PE can be captured by this model [31]. Afterwards this model was mainly used to investigate the nature of thermoacoustic waves $[18,19,38]$. This model can give an accurate description 
of the PE but involves complicated and heavy computations.

Boukari et al. [3] presented a thermodynamic model to describe the PE under zero gravity condition. They assumed that there is no velocity field and the pressure in the cell is only the function of time, since pressure equilibrium is achieved by sound propagation during a very short time period. This non-flow model has a relatively simple form and has been widely used by researchers to validate the experimental data [37] and explore the nature of PE [12, 33, 34].

In the present study, this thermodynamic model is chosen and the spherical coordinate is applied to describe and solve physical quantities. Because there is no velocity, and the pressure is space-independent, and the temperature and density change along the radius of the cell spatially, this problem can be simplified into one-dimensional form. The thermodynamic model in one-dimensional spherical coordinate system can be derived as following:

$$
\frac{\partial T}{\partial t}=\frac{1}{\rho C_{P}} \frac{1}{(R-x)^{2}} \frac{\partial}{\partial x}\left(\lambda(R-x)^{2} \frac{\partial T}{\partial x}\right)+\left(1-\frac{1}{\gamma}\right)\left(\frac{\partial T}{\partial P}\right)_{\rho} \frac{\partial P}{\partial t},
$$

$$
\frac{\partial P}{\partial t}=\frac{\int_{0}^{R}(R-x)^{2} \rho \alpha_{P}(\partial T / \partial t) \mathrm{d} x}{\int_{0}^{R}(R-x)^{2} \rho \kappa_{T} \mathrm{~d} x},
$$

with $T$ the temperature, $\rho$ the density, $P$ the pressure, $t$ the time, $\lambda$ the thermal conductivity, $C_{P}$ the isobaric specific heat, $\gamma=C_{P} / C_{V}$ the specific heat ratio, $\alpha_{P}$ the isobaric expansion coefficient and $\kappa_{T}$ the isothermal compressibility. This onedimensional thermodynamic model was also derived and used in Straub's study [12]. The spatial coordinate $x$ is along the radial direction with $x=0$ at the sample wall and $x=R$ at the center of the cell (shown in Fig.1) [12]. Eq.(4) is derived based on the energy conservation equation. The second term on the righthand side of Eq.(4) is the heat generation rate and is responsible for an isentropic 
temperature change in the bulk fluid. For an incompressible fluid $\gamma=1$, this term equals zero and Eq.(4) becomes the Fourier equation. Eq.(5) is derived based on both the equation of state and mass conservation and can be used to calculate the pressure derivative in Eq.(4).

Eq.(4) and Eq.(5) are solved by an explicit finite difference method using a central difference scheme for the spatial discretization. An iteration procedure is used to determine the pressure for each time step, in which the temperature in the whole fluid volume is calculated for some trial values of $P$. The $\rho=f(P, T)$ relations and thermodynamic properties of near-critical fluids are obtained from the NIST standard database [32]. Every parameter is the function of both temperature and pressure. The convergence criterion for $P$ is

$$
\frac{\left|P^{i+1}-P^{i}\right|}{P^{i}} \leqslant 10^{-5} .
$$

Masuda et al. [33] pointed out that an accurate solution to a near-critical fluid PE problem shall be obtained when the grid in the very thin boundary layer is sufficiently fine. But a large number of grid nodes means a heavy calculation and long computing time. Taking into consideration both the computing time and accuracy, nonuniform grids are carefully generated in the calculation domain of the present study. The minimum grid spacing $\Delta x$ is $0.005 \mathrm{~mm}$ at the boundary $(x=$ 0), which is half of Shen's [18] and Hasan's [38] grid spacing in their studies, and the spacing ratio for adjacent grid nodes is 1.001 along the direction of increasing $x$ (with around 1000 mesh grids for the $R=10 \mathrm{~mm}$ case).

For explicit finite difference method, the time step should be small enough to have numerical stability. So we test a selection of time steps, ranging from $0.0001 \mathrm{~s}$ to $0.01 \mathrm{~s}$. At last, a time step $0.001 \mathrm{~s}$ is adopted in most cases. In addition, a numerical algorithm based on implicit finite difference method is also 
established and operates under the same condition. The resulting temperature responses from two methods are almost identical. Notably, the approximation of space-independent pressure is only supported when the timescales of interest are much larger than $L / v_{a}$ [3], where $v_{a}$ is the speed of sound and $L$ is characteristic length, and all cases throughout this study satisfy this constraint $\left(L / v_{a} \sim 10^{-5} \mathrm{~s}\right)$.

\subsection{Verification of the numerical scheme}

In this section, the numerical prediction of the $\mathrm{PE}$ in near-critical $\mathrm{SF}_{6}$ based on the present numerical method is compared with an experimental measurement. In the Ref.[12], Straub et al. measured dynamic temperature propagation in a spherical cell filled with near-critical $\mathrm{SF}_{6}$ using four thermistors monitored at three different radial positions in the fluid and the cell wall. They pasted the heater (a wound wire) on the outer surface of the cell along a great circle and the surface can be treated as an almost isothermal heating area. Thirty nine cases of different initial conditions were measured and the existences of PE were confirmed. Besides, numerical simulations were performed by iteratively solving the energy equation to test and verify their measurements and good agreements were obtained.

In order to verify the numerical scheme presented in the present study, we carried out a simulation based on the same initial $\left(T_{0}=T_{c}+0.1 \mathrm{~K}, \rho_{0}=\rho_{c}\right)$ and boundary conditions with the measurements. Figure 2 shows the comparison between the computation in the present study and the measurements. It can be seen that the calculated bulk temperature change agrees well with experimental result. The average deviation is only $0.24 \mathrm{mK}$ and average relative deviation is $4.81 \%$. Thus the numerical scheme developed in this paper is acceptable and proved being effective to simulate the PE problem. 


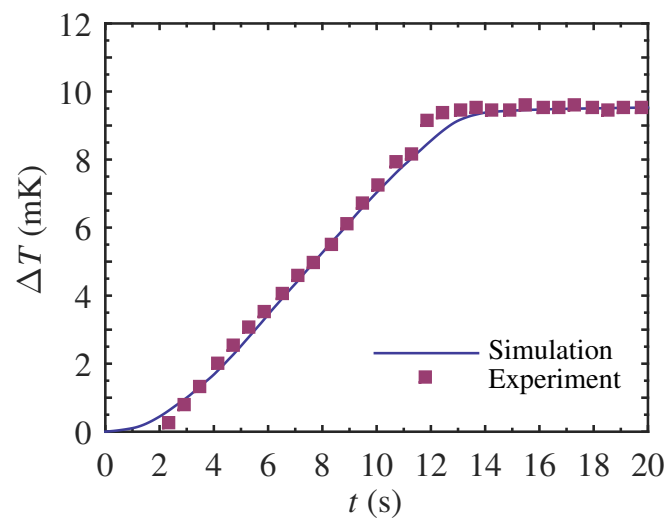

Figure 2: Comparison between the calculated bulk temperature change $(\Delta T)$ and measurements in the time region $0<t<20 \mathrm{~s}$ at $x=8.4 \mathrm{~mm}$ of a spherical cell $(R=9.6 \mathrm{~mm})$ filled with near-critical $\mathrm{SF}_{6}$. Solid line, calculated result; $\boldsymbol{\square}$, experimental data from [12].

\section{Definitions of physical quantities}

In this section, some important physical quantities are introduced, which are used in the present study to describe the PE problem.

Firstly, the timescale of the PE for a spherical enclosure is derived following a thermodynamic approach proposed by Onuki et al. [2]. It is convenient to assume that a small quantity of heat, $\Delta Q$, is added uniformly to a spherical enclosure. Then there forms a thin thermal boundary layer with a thickness $\delta$ near the wall. The whole volume can be divided into two parts, namely the thermal boundary layer and the bulk fluid, whose volumes are

$$
V_{1} \approx 4 \pi R^{2} \delta, V_{2} \approx \frac{4}{3} \pi R^{3}
$$

respectively. Under the influence of $\Delta Q$, the temperature and volume in $V_{1}$ will change by

$$
\Delta T_{1}=\frac{\Delta Q}{V_{1} C_{p}}, \Delta V_{1}=\alpha_{p} V_{1} \Delta T_{1}
$$


respectively, in which the approximation of a constant pressure process is applied. Because the total volume is constant, the expansion causes an adiabatic contraction of the bulk fluid $V_{2}$ by $\Delta V_{1}$. According to the adiabatic coefficient, $V(\partial T / \partial V)_{S}=-(\gamma-1) / \alpha_{P}$, the temperature of the bulk fluid will increase by

$$
\Delta T_{2}=\left(\frac{\partial T}{\partial V}\right)_{S}\left(-\Delta V_{1}\right)=\frac{\gamma-1}{\alpha_{P}} \Delta V_{1}
$$

The thermal equilibrium is reached when $\Delta T_{1}=\Delta T_{2}$. Along with Eq.(7-9), it leads to

$$
\delta=\frac{R / 3}{\gamma-1}
$$

Since $\delta=L /(\gamma-1)$, the characteristic length for a spherical cell is $L=R / 3$. Since diffusion dominates the formation of a thermal boundary layer, the timescale of the $\mathrm{PE}$ is

$$
t_{P E}=\frac{\delta^{2}}{D_{T}}=\frac{(R / 3)^{2}}{D_{T}(\gamma-1)^{2}},
$$

where $D_{T}$ is thermal diffusivity.

In order to measure temperature equilibrium capability of the PE, the temperature efficiency $\xi_{T}$ is defined as

$$
\xi_{T}=\frac{\Delta T_{\text {bulk }}}{\Delta T_{\text {bulk,ideal }}},
$$

where $\Delta T_{\text {bulk }}$ is the real bulk temperature increase and $\Delta T_{\text {bulk,ideal }}$ is the temperature which would be reached if the energy uniformly distributes in the fluid by heat diffusion. Physically, it implies the temperature improving ability ratio of the PE to pure heat diffusion. $\Delta T_{\text {bulk,ideal }}$ can be expressed as

$$
\Delta T_{\text {bulk,ideal }}=\frac{E_{\text {total }}}{m C_{V}}
$$


where $m$ is the mass of the fluid in the cell and $C_{V}$ is the specific heat capacity at constant volume. $E_{\text {total }}$ is calculated by

$$
E_{\text {total }}=\int A Q d t=\left.\int A \lambda \frac{\mathrm{d} T}{\mathrm{~d} x}\right|_{x=0} \mathrm{~d} t
$$

where $A=4 \pi R^{2}$ is surface area of the cell.

\section{Results and discussion}

\subsection{Effects of the specific heat ratio on the PE}

Numerical simulations were carried out for eight near-critical fluids to find out the influences of the specific heat ratio on the PE. The cell radius $R$ is $0.01 \mathrm{~m}$. Initially, the fluids are at their critical isochore $\left(\rho_{0}=\rho_{c}\right)$ and at the same initial reduced temperature $(\varepsilon=0.0005)$. The reduced temperature is a nondimensional parameter which is nondimensionalized by critical temperature. Inspired by the reduced temperature, we define a nondimensional boundary temperature rise as $\Delta T_{\text {wall }} / T_{c}$ and set it equals 0.00001 for all fluids. The initial and boundary conditions in this section are summarized in Eq.(1-3) and $R=0.01 \mathrm{~m}$. The chosen fluids and their detailed critical parameters, initial and boundary conditions are shown in Table 1.

The chosen fluids are representative since their critical parameters cover a large scope (the critical temperature varies form about $5.2 \mathrm{~K}$ to $647.1 \mathrm{~K}$ and the critical pressure varies form 0.23 $\mathrm{MPa}$ to 22.06 $\mathrm{MPa}$ ). As a result, it can be seen from Table 1 that the dimensional boundary temperature change $\Delta T_{\text {wall }}$ varies widely. The nondimensional boundary temperature rise change $\Delta T_{\text {wall }} / T_{c}=$ 0.00001 is negligible compared with $\varepsilon=0.0005$. The main purpose is to ensure the physical properties do not change dramatically during the PE process. 
Table 1: Critical parameters, initial and boundary conditions for eight fluids.

\begin{tabular}{lccccc}
\hline Fluid & $T_{c}(\mathrm{~K})$ & $P_{c}(\mathrm{MPa})$ & $T_{0}(\mathrm{~K})$ & $\rho_{0}\left(\mathrm{~m}^{3} / \mathrm{kg}\right)$ & $\Delta T_{\text {wall }}(\mathrm{mK})$ \\
\hline $\mathrm{He}$ & 5.1953 & 0.228 & 5.1979 & 72.567 & 0.052 \\
$\mathrm{H}_{2}$ & 33.145 & 1.296 & 33.162 & 31.262 & 0.331 \\
$\mathrm{Xe}$ & 289.73 & 5.842 & 289.88 & 1102.9 & 2.897 \\
$\mathrm{O}_{2}$ & 145.58 & 5.043 & 145.66 & 436.14 & 1.546 \\
$\mathrm{~N}_{2}$ & 126.19 & 3.396 & 126.25 & 313.30 & 1.262 \\
$\mathrm{H}_{2} \mathrm{O}$ & 647.10 & 22.06 & 647.42 & 322.00 & 6.471 \\
$\mathrm{CO}_{2}$ & 304.13 & 7.377 & 304.28 & 467.60 & 3.041 \\
$\mathrm{SF}_{6}$ & 318.72 & 3.755 & 318.88 & 742.30 & 3.187 \\
\hline
\end{tabular}

Table 2 presents the PE timescale and thermophysical properties of these nearcritical fluids under initial conditions. The fluids are listed in the order of descending $\gamma$. As we can see, the data in each column of Table 2 differs greatly. For example, the $\gamma$ of $\mathrm{He}$ is about four times that of $\mathrm{SF}_{6}$, and the $t_{P E}$ of $\mathrm{CO}_{2}$ is about three times that of $\mathrm{H}_{2} \mathrm{O}$.

In this section, the temperature, time and length are nondimensionalized by the critical temperature $T_{c}$, the PE timescale $t_{P E}$ and the cell radius $R$ respectively to make different fluids be able to be compared with each other, which leads

$$
T^{\prime}=\frac{T}{T_{c}}, t^{\prime}=\frac{t}{t_{P E}}, x^{\prime}=\frac{x}{R}
$$

As will be seen later, after nondimensionalization, results show general rules between the specific heat ratio and the PE. In the studies of the effects of characteristic length and boundary conditions on the PE, only one near-critical fluid is involved, so it is not necessary to nondimensionalize variables. 
Table 2: The PE timescale and thermophysical properties under initial conditions.

\begin{tabular}{lcccc}
\hline Fluid & $\gamma$ & $t_{P E}(\mathrm{~s})$ & $D_{T}\left(\mathrm{~m}^{2} / \mathrm{s}\right)$ & $\lambda(\mathrm{W} / \mathrm{m} \cdot \mathrm{K})$ \\
\hline $\mathrm{He}$ & 1398.8 & 0.0523 & $1.0868 \times 10^{-10}$ & 0.0374 \\
$\mathrm{H}_{2}$ & 1257.4 & 0.0361 & $1.9546 \times 10^{-10}$ & 0.0641 \\
$\mathrm{Xe}$ & 969.80 & 0.0262 & $4.5225 \times 10^{-10}$ & 0.1077 \\
$\mathrm{O}_{2}$ & 909.30 & 0.0491 & $2.7441 \times 10^{-10}$ & 0.1464 \\
$\mathrm{~N}_{2}$ & 793.92 & 0.0464 & $3.8049 \times 10^{-10}$ & 0.1766 \\
$\mathrm{H}_{2} \mathrm{O}$ & 687.18 & 0.0228 & $1.0348 \times 10^{-09}$ & 1.3992 \\
$\mathrm{CO}_{2}$ & 555.09 & 0.0677 & $5.3469 \times 10^{-10}$ & 0.3021 \\
$\mathrm{SF}_{6}$ & 361.03 & 0.0604 & $1.4187 \times 10^{-09}$ & 0.4000 \\
\hline
\end{tabular}

\subsubsection{Bulk temperature evolutions}

Displayed in Fig.3 (a) are the nondimensional temperature evolutions at the cell center for the near-critical fluids. Under the influence of PE, the nondimensional bulk temperature increases rapidly for $t^{\prime}=0 \sim 5$. Besides, its increase rate reduces gradually with the nondimensional time. This is a sign of the weakening PE and this trend is identical for all fluids.

In order to show the differences more clearly, Fig.3 (b) presents spatial profiles of $\Delta T^{\prime}$ for the different fluids at $t^{\prime}=15$. It is clear that the nondimensional bulk temperature increase for these fluids are markedly different and can be ranked as follows:

$$
\mathrm{He}<\mathrm{H}_{2}<\mathrm{Xe}<\mathrm{O}_{2}<\mathrm{N}_{2}<\mathrm{H}_{2} \mathrm{O}<\mathrm{CO}_{2}<\mathrm{SF}_{6},
$$

which coincides with the descending order of $\gamma$ (see the second column in Table 2 ). That is, the larger the $\gamma$ is, the slower the nondimensional bulk temperature increases with the nondimensional time. This will be clarified later in this section. 

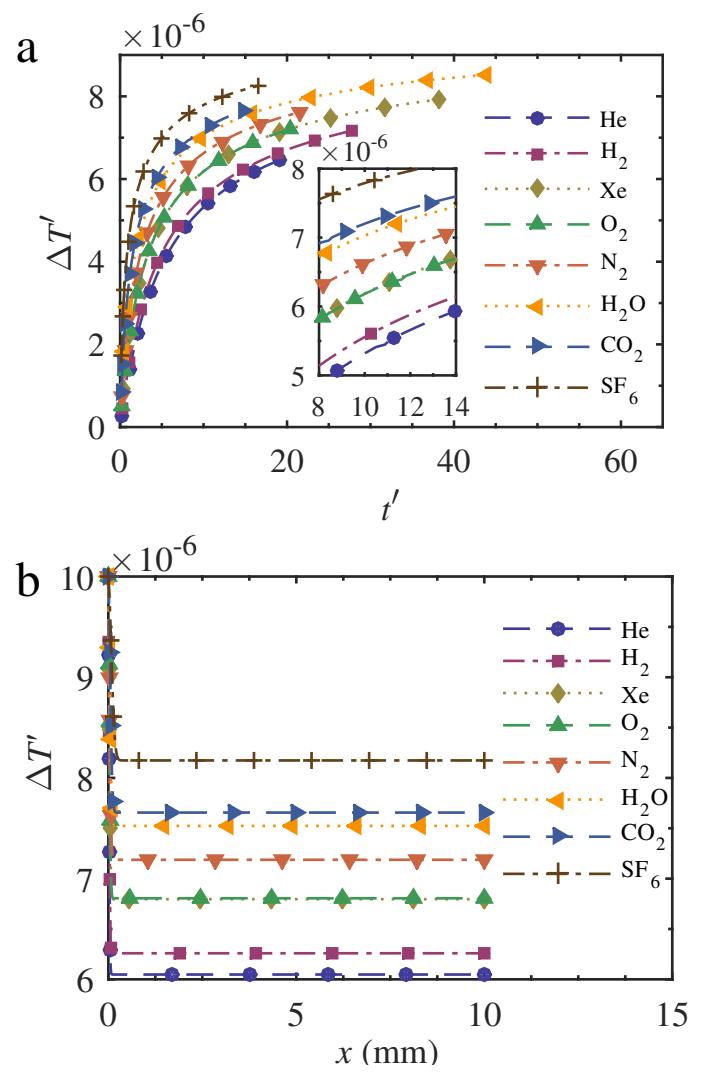

Figure 3: (a) Nondimensional temperature rise versus nondimensional time for fluids at $x=R$. (b) Spatial profiles of $\Delta T^{\prime}$ for fluids at $t^{\prime}=15$, where $\Delta T^{\prime}=\Delta T / T_{c}=\left(T-T_{0}\right) / T_{c}$ and $t^{\prime}=t / t_{P E} \cdot \bullet$, $\mathrm{He} ; \boldsymbol{\square}, \mathrm{H}_{2} ; \triangleleft, \mathrm{Xe} ; \boldsymbol{\Delta}, \mathrm{O}_{2}, \mathbf{\nabla}, \mathrm{N}_{2} ; \triangleleft, \mathrm{H}_{2} \mathrm{O} ; \bullet, \mathrm{CO}_{2} ;+, \mathrm{SF}_{6}$. 
Under small thermal disturbance, the physical properties of a fluid can be assumed as constant, which means they are time-independent and space-independent. Note that this approximation is justified under small thermal disturbance. Then Eq.(4) and Eq.(5) can be simplified into the following form

$$
\begin{aligned}
\frac{\partial T}{\partial t}= & \frac{D_{T}}{(R-x)^{2}} \frac{\partial}{\partial x}\left((R-x)^{2} \frac{\partial T}{\partial x}\right)+ \\
& \left(1-\frac{1}{\gamma}\right) \frac{3}{R^{3}} \int_{0}^{R}(R-x)^{2} \frac{\partial T}{\partial t} \mathrm{~d} x .
\end{aligned}
$$

Define nondimensional variables as Eq.(15), and Eq.(17) is transformed into nondimensional form

$$
\begin{aligned}
\frac{\partial T^{\prime}}{\partial t^{\prime}}= & \frac{1}{9(\gamma-1)^{2}} \frac{1}{\left(1-x^{\prime}\right)^{2}} \frac{\partial}{\partial x^{\prime}}\left(\left(1-x^{\prime}\right)^{2} \frac{\partial T^{\prime}}{\partial x^{\prime}}\right)+ \\
& 3\left(1-\frac{1}{\gamma}\right) \int_{0}^{1}\left(1-x^{\prime}\right)^{2} \frac{\partial T^{\prime}}{\partial t^{\prime}} \mathrm{d} x^{\prime}
\end{aligned}
$$

It is shown that the specific heat ratio is the unique physical property in Eq.(18), which means all the others being equal, the specific heat ratio is the only factor that influence the nondimensional bulk temperature increase for the different nearcritical fluids under small thermal disturbance. As shown in Eq.(18), $1 /(\gamma-1)^{2}$ and $(1-1 / \gamma)$ are coefficients in the right-hand side of Eq.(18). The effects of $\gamma$ on the coefficients can be illustrated by the following equations:

$$
\frac{1 /\left(\gamma_{\mathrm{He}}-1\right)^{2}}{1 /\left(\gamma_{\mathrm{SF}_{6}}-1\right)^{2}}=0.0663, \frac{1-1 / \gamma_{\mathrm{He}}}{1-1 / \gamma_{\mathrm{SF}_{6}}}=1.0021,
$$

where $\gamma_{\mathrm{He}}=1398.8, \gamma_{\mathrm{SF}_{6}}=361.03$. It can be seen from Eq.(19) that the first coefficient $1 /(\gamma-1)^{2}$ is more sensitive to the variation of $\gamma$, and it is appropriate to suppose $\gamma$ has no effect on the second coefficient $(1-1 / \gamma)$. So the increase of $\gamma$ will reduce the first term in the right-hand side of Eq.(18) considerably, and cause the decrease of the left-hand term $\partial T^{\prime} / \partial t^{\prime}$ further. However, the second term in 
the right-hand side of Eq.(18) is responsible for the bulk temperature change and is strongly coupled with $\partial T^{\prime} / \partial t^{\prime}$. Thus the increase of $\gamma$ will further diminish the nondimensional bulk temperature change.

Note that Masuda et al. [33] and Mao et al. [34] simulated the PE in nearcritical $\mathrm{H}_{2} \mathrm{O}$. They compared the bulk temperature change at the same time and concluded that the influence of the PE is stronger as the $\gamma$ is larger. However, it seemingly contradict with the conclusion presented here based on simulations of eight near-critical fluids. In fact, there is no contradiction and the type of time chosen to compare ( $t^{\prime}$ or $\left.t\right)$ is the key reason to this observed phenomenon. For a single near-critical fluid, a larger $\gamma$ means a smaller $t_{P E}[34]$ and consequently means a larger nondimensional time $t^{\prime}$ at the same normal time $t$. As a result, when nondimensional bulk temperature increase at the same nondimensional time $t^{\prime}$ is compared, it is found that a larger $\gamma$ means a slower nondimensional bulk temperature increase; but when the comparison is made for dimensional bulk temperature increase at the same normal time $t$, it is found that a larger $\gamma$ means a faster bulk temperature increase. Besides, Masuda et al. [33] pointed out for a single nearcritical fluid, a larger $\gamma$ means a smaller thermal diffusivity $D_{T}$. But it can be concluded from Table 2 that if more than one near-critical fluids are involved, the relationship among $\gamma$ and $D_{T}$ can't be simply established. For example, the $\gamma$ of $\mathrm{H}_{2} \mathrm{O}$ is larger than that of $\mathrm{CO}_{2}$, but the $D_{T}$ of $\mathrm{H}_{2} \mathrm{O}$ is also larger than that of $\mathrm{CO}_{2}$.

In order to verify above analysis, numerical simulations are preformed for near-critical $\mathrm{CO}_{2}$ under two typical reduced temperature. The PE timescale and thermophysical properties are shown in Table 3, and the calculated dimensional and nondimensional bulk temperature responses are shown in Fig.4. It is shown in Table 3 and Fig.4(a) that near the critical point, the specific heat ratio of $\mathrm{CO}_{2}$ in- 
creases and bulk temperature change accelerates, which is consistent with Masuda et al. [33]. But as shown in Fig.4(b), nondimensional variables show opposite trend, which is consistent with the conclusions in the present study.

Table 3: The PE timescale and thermophysical properties for near-critical $\mathrm{CO}_{2}$ under different reduced temperature.

\begin{tabular}{ccccc}
\hline$\varepsilon$ & $\gamma$ & $t_{P E}(\mathrm{~s})$ & $D_{T}\left(\mathrm{~m}^{2} / \mathrm{s}\right)$ & $\lambda(\mathrm{W} / \mathrm{m} \cdot \mathrm{K})$ \\
\hline 0.00100 & 286.56 & 0.1643 & $8.2935 \times 10^{-10}$ & 0.2247 \\
0.00329 & 90.993 & 0.7128 & $1.9246 \times 10^{-09}$ & 0.1388 \\
\hline
\end{tabular}

\subsubsection{Temperature efficiency evolutions}

Figure 5 presents variations of the temperature efficiency with the nondimensional time for the different near-critical fluids. It is seen that there are three obvious trends related to this temperature efficiency.

Firstly, the observed temperature efficiencies are all higher than 0.995, which indicates the PE is extremely efficient in terms of temperature equilibrium. This has also been reported by Garrabos et al. [14] and was attributed to the fact that the internal energy of near-critical fluid is very sensitive to density variations. The small mass flux getting into the bulk at the hot boundary layer border causes the density change precisely [14].

Secondly, the temperature efficiencies for the different fluids are unequal, but the observed maximum difference is only 0.002 . This maybe because the temperature efficiency is related to the particular nature of near-critical fluids. The initial reduced temperature for the eight fluids are identical, so the nature of near-critical fluids are nearly the same. Moreover, the temperature efficiencies almost do not 

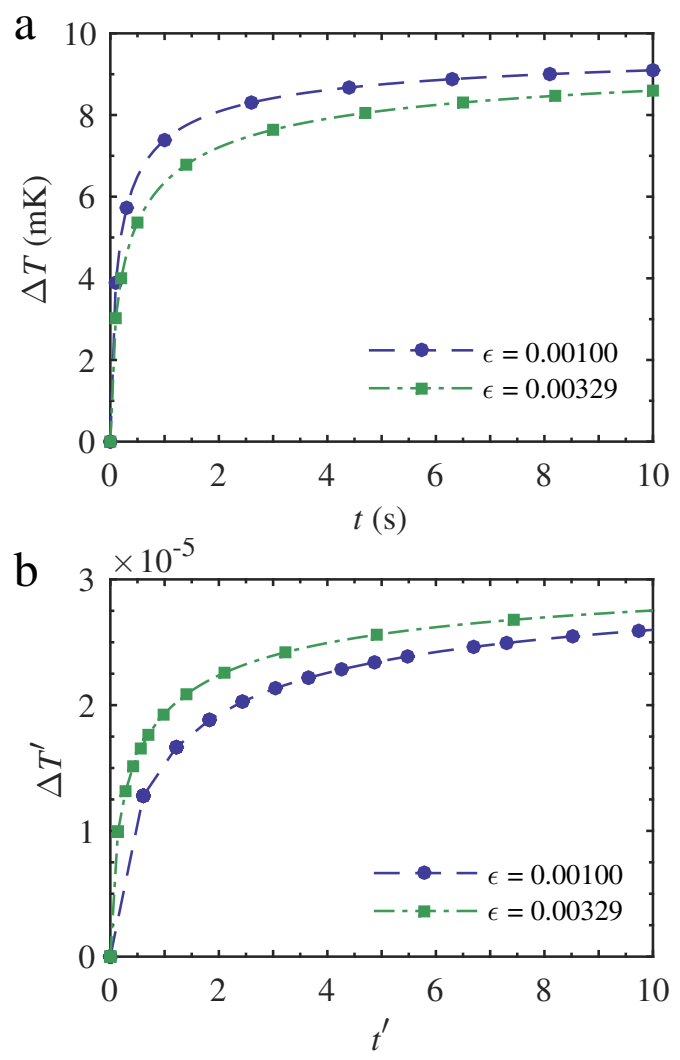

Figure 4: (a) Dimensional and (b) nondimensional bulk temperature responses for near-critical $\mathrm{CO}_{2}$ and different reduced temperature, where $\Delta T^{\prime}=\Delta T / T_{c}=\left(T-T_{0}\right) / T_{c}$ and $t^{\prime}=t / t_{P E} ; \bullet$, $\varepsilon=0.001, \boldsymbol{\square}, \varepsilon=0.00329$. 


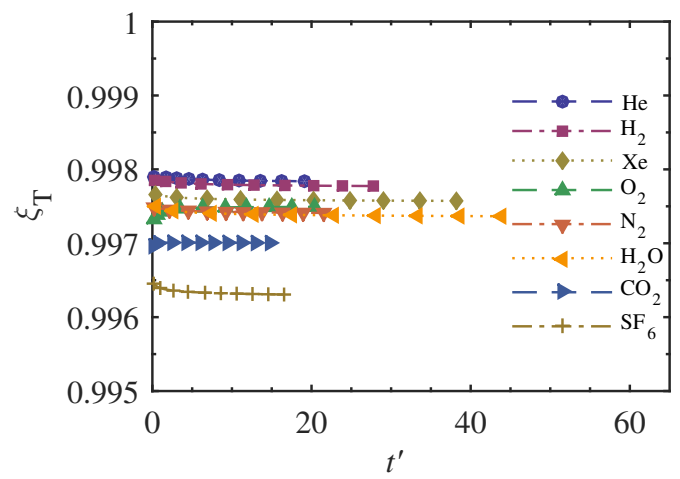

Figure 5: Temperature efficiency $\xi_{T}$ versus nondimensional time $t^{\prime}=t / t_{P E}$ for different near-

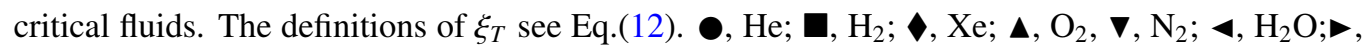
$\mathrm{CO}_{2} ;+, \mathrm{SF}_{6}$.

change with the nondimensional time. This maybe because the imposed thermal disturbance is too small to change the initial reduced temperature during the PE process.

Thirdly, the specific heat ratio simply reflects the temperature efficiency, which shows the larger the specific heat ratio, the higher the temperature efficiency. The relative temperature efficiency of the eight near-critical fluids are as follows:

$$
\mathrm{He}>\mathrm{H}_{2}>\mathrm{Xe}>\mathrm{O}_{2}>\mathrm{N}_{2}>\mathrm{H}_{2} \mathrm{O}>\mathrm{CO}_{2}>\mathrm{SF}_{6} \text {, }
$$

which coincides with the ascending order of $\gamma$ (see Table 2). Thus in terms of temperature equilibrium, the PE is strong and capable as the specific heat ratio becomes larger. This maybe because the underlying mechanisms of the densitysensitive internal energy and the enlarged specific heat ratio are closely related.

In this section, a nondimensionalization method $\left(T_{c}, t_{P E}\right.$, and $R$ for temperature, time, and length respectively) is developed and numerical simulations of the PE for eight near-critical fluids are preformed. Results show the specific heat 
ratio is an efficient indicator of the $\mathrm{PE}$, which is negatively corrected with the increase rate of nondimensional bulk temeprature and positively correlated with the temperature efficiency.

\subsection{Effects of the characteristic length on the PE}

As mentioned in Section 3, the characteristic length, denoted as $L$, equals $R / 3$ for a spherical cell. Moreover, the heating-surface-area-to-volume ratio for the physical model shown in Fig. 1 is

$$
\frac{4 \pi R^{2}}{\frac{4}{3} \pi R^{3}}=\frac{3}{R},
$$

which is exactly the reciprocal of the characteristic length. So physically, the characteristic length is the ratio of the cell volume to the heating surface area and is negatively correlated with the thermal disturbance obtained by per unit volume.

According to the analytical solution given by Onuki et al. [39], Straub et al. [12] noted that with an decreasing characteristic length, the bulk temperature increase accelerates, which is in agreement with its physical meaning. In this section, a series of numerical simulations are carried out to elaborate how the $L$ influences the PE. Three spherical cells $(R=0.1,0.01$ and $0.001 \mathrm{~m}, L=3.33 \times$ $10^{-2}, 3.33 \times 10^{-3}$ and $3.33 \times 10^{-4} \mathrm{~m}$ respectively) filled with near-critical $\mathrm{CO}_{2}$ $\left(T_{c}=304.13 \mathrm{~K}, \rho_{c}=467.6 \mathrm{~kg} / \mathrm{m}^{3}\right)$ are used in the present study. The initial and boundary conditions are shown in Eq.(1-3), with $\varepsilon=0.00033, \Delta T_{\text {wall }}=10 \mathrm{mK}$.

\subsubsection{Bulk temperature evolutions}

Figure 6 shows bulk temperature evolutions for the characteristic lengths. It is shown that the bulk temperature increases rapidly at initial stage, and the increase 

observed and explained in Section 4.1.1.

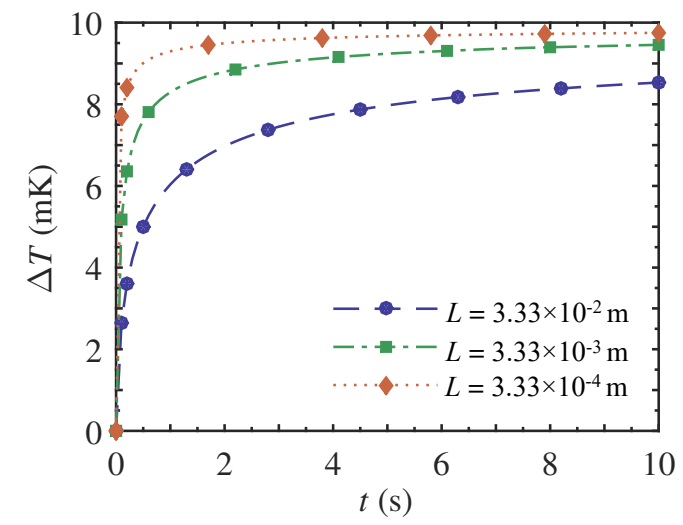

Figure 6: Temperature changes at $x=R$ versus $t$ for the different characteristic lengths. $L=3.33 \times 10^{-2} \mathrm{~m} ; \boldsymbol{\square}, L=3.33 \times 10^{-3} \mathrm{~m} ; \boldsymbol{\diamond}, L=3.33 \times 10^{-4} \mathrm{~m}$.

rate reduces gradually. They are typical bulk temperature responses and have been

Moreover, it is observed that the shorter the characteristic length is, the faster the bulk temperature increases. For example, the $L$ changing from $3.33 \times 10^{-2} \mathrm{~m}$ to $3.33 \times 10^{-4} \mathrm{~m}$, the time for the bulk temperature increasing by $8.5 \mathrm{mK}$ reduces from $10 \mathrm{~s}$ to $0.2 \mathrm{~s}$. This can be explained by the fact that for a shorter $L$, the propagating period of thermoacoustic waves is shorter, which will help to enhance the bulk temperature increase. This law has also been pointed out by Straub et al. [12], based on the analytical solution given by Onuki et al. [39].

\subsubsection{Boundary heat flux evolutions}

Shown in Fig.7 are time variations of the boundary heat flux for the characteristic lengths. Three obvious characteristics concerning the boundary heat flux can be seen from the computed curves.

Firstly, the heat flux approaches zero rapidly. It is because the temperature of the hot boundary layer increases and reaches boundary temperature. 


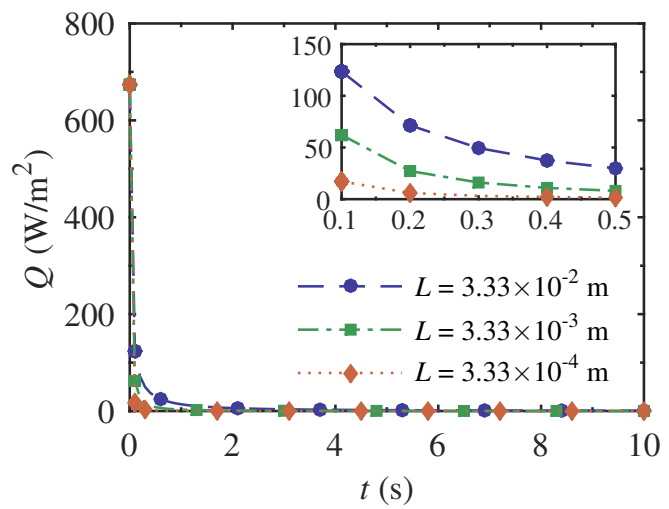

Figure 7: Temporal variations of the heat flux at the boundary for the different characteristic lengths. Inset: the enlarged portion for $t=0.1 \sim 0.5 \mathrm{~s}$.,$L=3.33 \times 10^{-2} \mathrm{~m} ; \boldsymbol{\square}, L=3.33 \times 10^{-3}$ $\mathrm{m} ; \bullet, L=3.33 \times 10^{-4} \mathrm{~m}$.

Secondly, the shorter the $L$ is, the faster the heat flux falls. This is because for a shorter $L$, the bulk temperature is higher and the PE is weaker at the same time. Thirdly, the shorter the $L$ is, the less heat is sent into the fluid. This is because a decreasing $L$ leads to a decreasing surface area and boundary heat flux, both of which will reduce the total heat sent into the fluid. Calculated results show, the $L$ changing from $3.33 \times 10^{-2} \mathrm{~m}$ to $3.33 \times 10^{-4} \mathrm{~m}$, the total heat sent into the fluid decreases by six order of magnitude.

\subsubsection{Temperature efficiency evolutions}

Figure 8 shows time variations of the temperature efficiency for the characteristic lengths. One can see the high and barely changing temperature efficiency, which has been reported and explained in Section 4.1.2. Besides, it is seen that the temperature efficiency is higher for a larger $L$. This maybe because for a shorter $L$, less heat is transferred into the bulk fluid owing to the smaller bulk fluid volume. That is to say, a larger part of energy is stored in the hot boundary layer and the 
temperature efficiency is low.

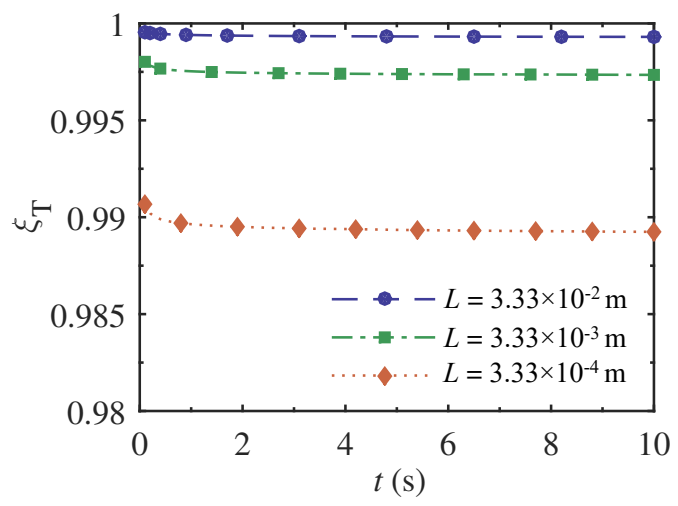

Figure 8: Temperature efficiency evolutions for the different characteristic lengths. $\bullet, L=3.33 \times$ $10^{-2} \mathrm{~m} ; \mathbf{\square}, L=3.33 \times 10^{-3} \mathrm{~m} ; \boldsymbol{\nabla}, L=3.33 \times 10^{-4} \mathrm{~m}$.

\subsection{Effects of the boundary condition on the PE}

The boundary condition is an important factor influencing the PE. Shen and Zhang studied the thermoacoustic waves emission, propagation and reflection under different temperature boundary conditions by solving the governing hydrodynamic equations [18]. Besides, they studied the thermoacoustic waves emission under internal and boundary heat flux conditions [19]. Hasan and Farouk also studied the thermoacoustic waves generation under different boundary heating rates using a real-fluid CFD model [20]. The thermodynamic model can reveal problems from a macroscopic view but it is not be adopted before to investigate effects of the boundary condition on the PE.

In this section, several Dirichlet boundary conditions are designed to study the effects of boundary conditions on the PE, in which the boundary temperature is known. A spherical cell $(R=0.01 \mathrm{~m})$ filled with near-critical $\mathrm{CO}_{2}\left(T_{c}=304.13 \mathrm{~K}\right.$, 
$\left.\rho_{c}=467.6 \mathrm{~kg} / \mathrm{m}^{3}\right)$ is considered in the presented analysis. The initial conditions are shown in Eq.(1) and Eq.(2), with $\varepsilon=0.00033$.

The boundary condition is the independent variable in this section. Taking into account the increase rate (the slope of the curve), four representative boundary conditions are designed, which have the same starting and end point (see Fig.9 and Table 4). The boundary conditions are named taking more of their geometry information into consideration. The variation laws for the increase rates are summarized as follows:

a. IR: the increase rate equals infinity when $t=0 \mathrm{~s}$ and equals zero when $t>0 \mathrm{~s}$;

b. LUQR: the increase rate reduces gradually from infinity to zero;

c. LR: the increase rate is constant and equals $1 \mathrm{mK} / \mathrm{s}$;

d. RLQR: the increase rate increases gradually from zero to infinity.

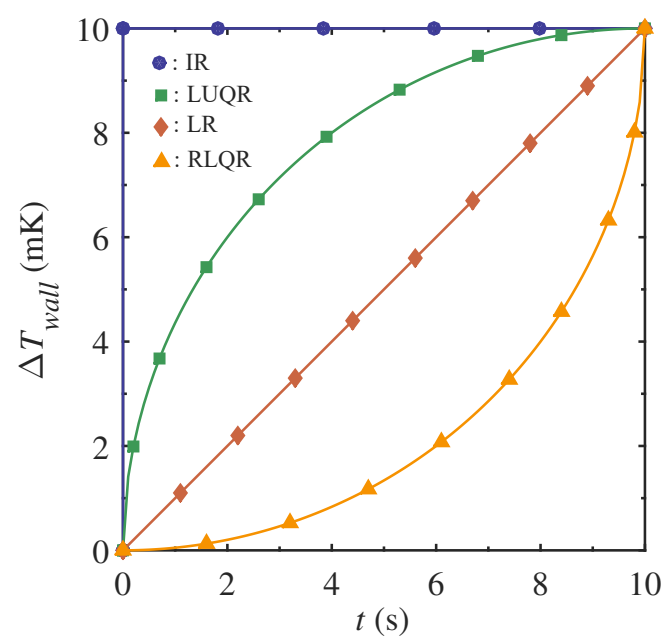

Figure 9: Temporal variations of change in boundary temperature. Implications of the abbreviations are listed in Table 4. ๑, IR; $\mathbf{\square}, \mathrm{LUQR} ;$, LR; $\mathbf{\Lambda}$, RLQR. 
Table 4: Detailed information of the boundary conditions.

\begin{tabular}{lll}
\hline Abbreviation & Full name & Mathematical expression \\
\hline IR & Impulsive Rise. & $\Delta T_{\text {wall }}=10(\mathrm{mK})$ \\
LUQR & Left Upper Qurant Rise. & $\Delta T_{\text {wall }}=\sqrt{20 t-t^{2}}(\mathrm{mK})$ \\
LR & Linear Rise. & $\Delta T_{\text {wall }}=t(\mathrm{mK})$ \\
RLQR & Right Lower Qurant Rise. & $\Delta T_{\text {wall }}=10-\sqrt{100-t^{2}}(\mathrm{mK})$ \\
\hline
\end{tabular}

They are representative because the increase rates cover the range from zero to infinity and all the possibility of variations. Note that we do not discuss the situation that the boundary temperature rise curve has negative slope, which may cause alternate hot piston effect and cool piston effect (CPE) [6] depending on the particular behaviors of the boundary temperature.

\subsubsection{Bulk temperature and pressure evolutions}

Shown in Fig.10 is the calculated responses of bulk temperature and pressure. Comparing the two figures, we can draw a conclusion that the bulk temperature and the pressure change synchronously. It is because the bulk temperature change is calculated from the pressure change. As illustrated in Fig.10 (a), the curves of the bulk temperature increase under the different boundary conditions are entirely different from each other. Thus the boundary condition is crucial in determining the bulk temperature response. Under IR, the boundary temperature suddenly increases by $10 \mathrm{mK}$ at $t=0 \mathrm{~s}$, and most of the bulk temperature change is accomplished during $0 \sim 2 \mathrm{~s}$. Compared with IR, under the other boundary conditions, the bulk temperature closely follows the boundary temperature. 

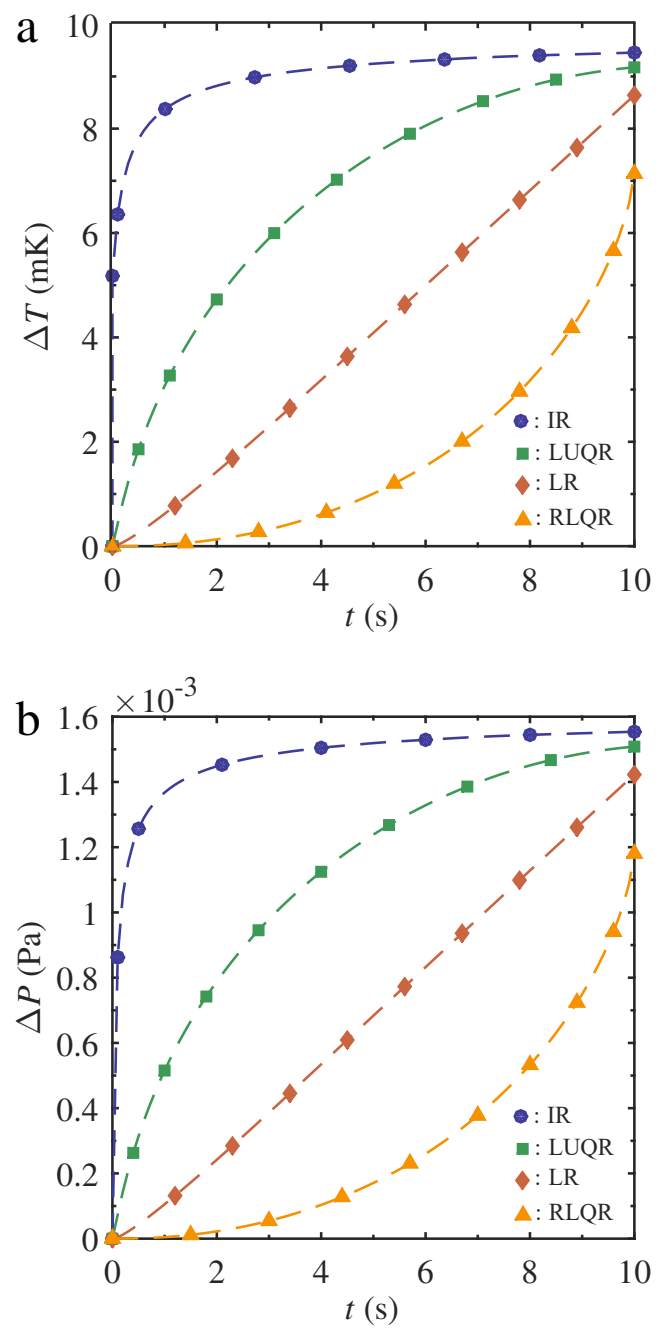

Figure 10: (a) Temporal variations of change in bulk temperature. (b) Temporal variations of change in pressure. $\bullet, 1 R ; \square, L U Q R ;, L R ; \mathbf{\Lambda}, \mathrm{RLQR}$. 
In order to evaluate boundary conditions, we define thermal impulse $I_{T}$ as

$$
I_{T}=\int \Delta T_{\text {wall }} \mathrm{d} t
$$

which indicates the thermal disturbance imposed to the fluid during a period of time, and equals the area enclosed by the curves, the line $\Delta T_{\text {wall }}=0 \mathrm{mK}$ and the vertical line $t=10 \mathrm{~s}$ (see Fig.9). The thermal impulse, bulk temperature increase, and total heat sent into the fluid at $t=10 \mathrm{~s}$ for the different boundary conditions are given in Table 5. It is shown that the higher the thermal impulse, the larger the bulk temperature increase. Thus the thermal impulse can be considered as an efficient parameter to evaluate boundary conditions, which is positively correlated with the bulk temperature increase.

Table 5: Thermal impulse, bulk temperature increase and total heat sent into the fluid at $t=10 \mathrm{~s}$.

\begin{tabular}{llll}
\hline Boundary Condition & $I_{T}(\mathrm{mK} \cdot \mathrm{s})$ & $\Delta T_{\text {bulk }}(\mathrm{mK})$ & $E_{\text {total }}(\mathrm{J})$ \\
\hline IR & 100 & 9.4564 & 0.0380 \\
LUQR & 79 & 9.1742 & 0.0369 \\
LR & 50 & 8.6394 & 0.0349 \\
RLQR & 21 & 7.0578 & 0.0291 \\
\hline
\end{tabular}

The relationship between the thermal impulse and bulk temperature increase is explained below. As mentioned before, the temperature difference between the boundary and bulk temperature is the driving force of PE. However, the thermal impulse is just the accumulation of the driving force during some time and consequently, should be positively correlated with the bulk temperature increase.

The relationship can also be explained by analogy with the impulse-momentum theorem in classical mechanics. A force can cause an object with mass to change 
its velocity; the impulse in classical mechanics is the integral of a force over the time interval during which it acts; the impulse-momentum theorem states that the impulse causes a change in momentum, which is to say the impulse is proportionate to the change in velocity. Analogously, a thermal disturbance can cause the bulk temperature change with the help of the PE; the thermal impulse is the integral of a thermal disturbance over the time interval during which it is imposed; therefore, the thermal impulse is positively correlated with the bulk temperature change. Thus, it is reasonable to call this kind of relationship as the impulsemomentum theorem in the PE. To be more specific, analogously, the force in PE is thermal disturbance, the impulse in PE is thermal impulse $I_{T}$, and the momentum in $\mathrm{PE}$ is the bulk temperature change.

Garrabos et al. [14] studied the PE under three heat pulses with identical total energy, and pointed out the bulk temperature responses eventually become identical over a period of time. Note as well in the present study, the thermal impulse is also positively correlated with the total heat sent into the fluid (see Table 5). So the differences in amount of heat sent into the fluid may be a reasonable explanation of the impulse-momentum theorem analogy.

\subsubsection{Boundary heat flux evolutions}

Figure 11 shows time variations of the boundary heat flux and the increase rate of boundary temperature for the different boundary conditions. There are obvious differences in the boundary heat flux curves, so the boundary condition has a strong influence on the boundary heat flux.

To be specific, the boundary heat flux is almost positively correlated with the increase rate of boundary temperature. This discipline can be concluded from comparing the curves in (a) and (b) respectively. According to Fourier's heat con- 

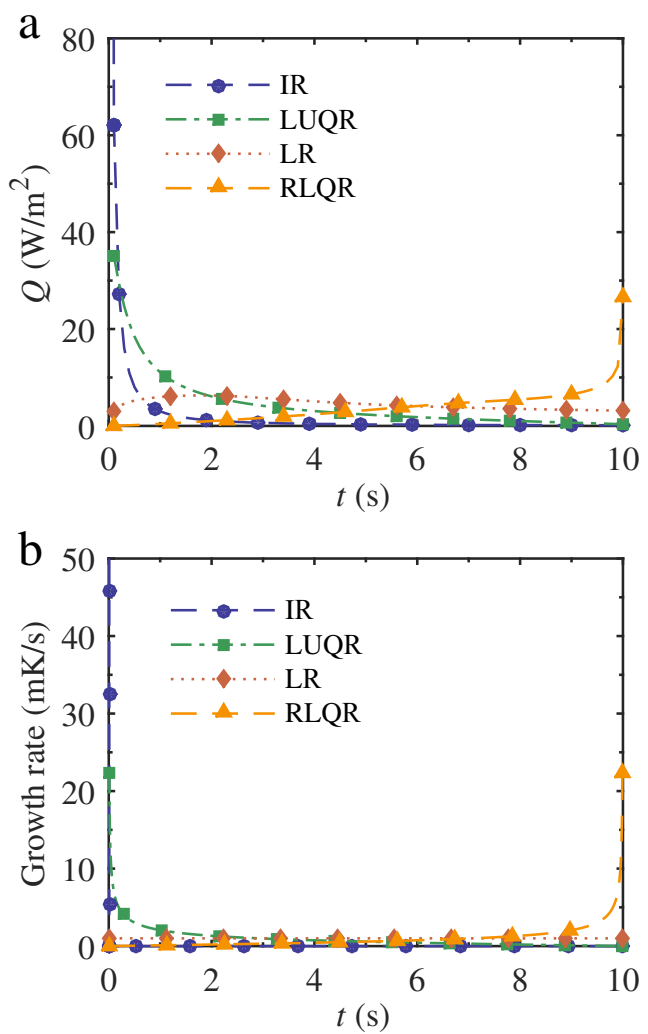

Figure 11: Temporal variations of (a) the boundary heat flux and (b) the increase rate of boundary temperature for the different boundary conditions. $\bullet, 1 R ; \mathbf{\square}, \mathrm{LUQR} ; \diamond, \mathrm{LR} ; \boldsymbol{\Delta}, \mathrm{RLQR}$. 


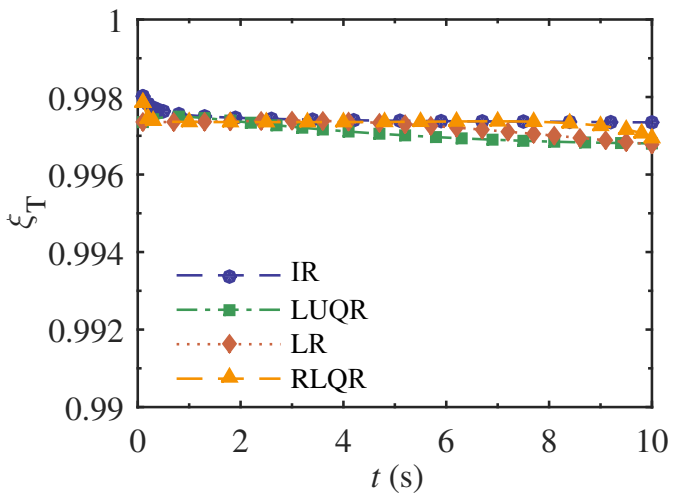

Figure 12: Temperature efficiency evolutions for the different boundary conditions. , $\mathrm{IR}$ LUQR; $\downarrow$ LR; $\Lambda$, RLQR. 


\section{Conclusion}

In this paper, a series of numerical explorations have been conducted into the piston effect in a spherical cell filled with near-critical fluid under zero gravity and small thermal disturbance conditions. Various factors that influence the piston effect $(\mathrm{PE})$, such as the specific heat ratio, the characteristic length and the boundary condition, are investigated by numerically solving the thermodynamic model.

The results exhibit the fast-responding bulk temperature, which is a sign of the PE, along with high and stable temperature efficiency, which is determined by the particular nature of near-critical fluids. A nondimensionalization method is developed to study the influences of the specific heat ratio on the PE based on simulations for eight near-critical fluids. Results show that the specific heat ratio is an efficient indicator of the PE, which is negatively correlated with the increase rate of nondimensional bulk temperature, but positively correlated with the temperature efficiency. The characteristic length weighs heavily upon the PE. Similarly, it is negatively correlated with the increase rate of bulk temperature, but positively correlated with the temperature efficiency. In addition, the newly defined thermal impulse can be considered as an efficient parameter to evaluate boundary conditions, which is positively correlated with the bulk temperature increase, but has slight influence on the temperature efficiency under small thermal disturbance. It is reasonable to call this kind of relationship as the impulse-momentum theorem in the PE.

In the present study, small thermal disturbance is only considered. Under strong thermal disturbance, the critical distance will be altered during the PE. As a result, the nature of near-critical fluids will change dramatically and the rules mentioned above may not well-established. Besides, the boundary temperature 
curve with negative slope is not studied, which may cause alternate hot and cool piston effect depending on the particular behaviors of the boundary temperature.

Both of the two topics are interesting and need to be studied furthermore.

\section{Acknowledgement}

The support of the National Science Foundation of China (No.51476001) is gratefully acknowledged.

\section{References}

[1] P. Carles, A brief review of the thermophysical properties of supercritical fluids, Journal of Supercritical Fluids 53 (1-3) (2010) 2-11.

[2] A. Onuki, H. Hao, R. A. Ferrell, Fast adiabatic equilibration in a singlecomponent fluid near the liquid-vapor critical point, Phys Rev A 41 (4) (1990) 2256-2259.

[3] H. Boukari, J. N. Shaumeyer, M. E. Briggs, R. W. Gammon, Critical speeding up in pure fluids, Phys Rev A 41 (4) (1990) 2260-2263.

[4] B. Zappoli, D. Bailly, Y. Garrabos, B. Le Neindre, P. Guenoun, D. Beysens, Anomalous heat transport by the piston effect in supercritical fluids under zero gravity, Phys Rev A 41 (4) (1990) 2264-2267.

[5] K. Nitsche, J. Straub, The critical "hump" of cv under microgravity: results from d1-spacelab experiment "wrmekapazitt", in: Proceedings of the 6th European Symposium on Material Sciences under Microgravity Conditions, ESA SP-256 (1987), pp. 109-116. 
[6] B. Zappoli, P. Carles, The thermo-acoustic nature of the critical speeding up, Vol. 14, Elsevier Masson, Issy-les-Moulineaux, FRANCE, 1995.

[7] B. Zappoli, P. Charles, Acoustic saturation of the critical speeding up, Physica D: Nonlinear Phenomena 89 (3-4) (1996) 381-394.

[8] B. Zappoli, P. Carles, S. Amiroudine, J. Ouazzani, Inversion of acoustic waves reflection rules in near critical pure fluids, Physics of Fluids (1994present) 7 (9) (1995) 2283-2287.

[9] P. Carles, The effect of bulk viscosity on temperature relaxation near the critical point, Physics of Fluids 10 (9) (1998) 2164-2178.

[10] D. Bailly, B. Zappoli, Hydrodynamic theory of density relaxation in nearcritical fluids, Physical Review E 62 (2) (2000) 2353-68.

[11] P. Guenoun, B. Khalil, D. Beysens, Y. Garrabos, F. Kammoun, B. Le Neindre, B. Zappoli, Thermal cycle around the critical point of carbon dioxide under reduced gravity, Physical Review E 47 (3) (1993) 1531-1540.

[12] J. Straub, L. Eicher, A. Haupt, Dynamic temperature propagation in a pure fluid near its critical point observed under microgravity during the german spacelab mission d-2, Physical Review E 51 (6) (1995) 5556-5563.

[13] F. Zhong, H. Meyer, Density equilibration near the liquid-vapor critical point of a pure fluid: Single phase $t>t_{c}$, Physical Review E 51 (4) (1995) 32233241 .

[14] Y. Garrabos, M. Bonetti, D. Beysens, F. Perrot, T. Frohlich, P. Carles, B. Zappoli, Relaxation of a supercritical fluid after a heat pulse in the absence of 
gravity effects: Theory and experiments, Physical Review E 57 (5) (1998) 5665-5681.

[15] Y. Miura, S. Yoshihara, M. Ohnishi, K. Honda, M. Matsumoto, J. Kawai, M. Ishikawa, H. Kobayashi, A. Onuki, High-speed observation of the piston effect near the gas-liquid critical point, Physical Review E 74 (1) (2006) 010101.

[16] P. Carles, Thermoacoustic waves near the liquid-vapor critical point, Physics of Fluids 18 (12) (2006) 126102.

[17] A. Onuki, Thermoacoustic effects in supercritical fluids near the critical point: Resonance, piston effect, and acoustic emission and reflection, Physical Review E 76 (6) (2007) 061126.

[18] B. Shen, P. Zhang, On the transition from thermoacoustic convection to diffusion in a near-critical fluid, International Journal of Heat and Mass Transfer 53 (21-22) (2010) 4832-4843.

[19] B. Shen, P. Zhang, Thermoacoustic waves along the critical isochore, Physical Review E 83 (1) (2011) 011115.

[20] N. Hasan, B. Farouk, Thermoacoustic transport in supercritical fluids at nearcritical and near-pseudo-critical states, Journal of Supercritical Fluids 68 (2012) 13-24.

[21] B. Zappoli, S. Amiroudine, P. Carles, J. Ouazzani, Thermoacoustic and buoyancy-driven transport in a square side-heated cavity filled with a nearcritical fluid, Journal of Fluid Mechanics 316 (1996) 53-72. 
[22] B. Zappoli, A. Jounet, S. Amiroudine, A. Mojtabi, Thermoacoustic heating and cooling in near-critical fluids in the presence of a thermal plume, Journal of Fluid Mechanics 388 (1999) 389-409.

[23] S. Amiroudine, P. Bontoux, P. Larroude, B. Gilly, B. Zappoli, Direct numerical simulation of instabilities in a two-dimensional near-critical fluid layer heated from below, Journal of Fluid Mechanics 442 (2001) 119-140.

[24] G. Accary, P. Bontoux, B. Zappoli, Turbulent rayleigh-benard convection in a near-critical fluid by three-dimensional direct numerical simulation, Journal of Fluid Mechanics 619 (2009) 127-145.

[25] B. Shen, P. Zhang, Rayleigh-benard convection in a supercritical fluid along its critical isochore in a shallow cavity, International Journal of Heat and Mass Transfer 55 (23-24) (2012) 7151-7165.

[26] B. Shen, P. Zhang, Three-dimensional thermoconvection from a nonuniformly heated plate near the liquid-vapor critical point, International Journal of Thermal Sciences 89 (2014) 136-153.

[27] S. Amiroudine, B. Zappoli, Piston-effect-induced thermal oscillations at the rayleigh-bénard threshold in supercritical h e 3, Physical review letters 90 (10) (2003) 105303.

[28] K. Boutrouft, S. Amiroudine, A. Ambari, Stability diagram and effect of initial density stratification for a two-layer system in a supercritical fluid, Physics of Fluids (1994-present) 18 (12) (2006) 124106.

[29] S. Amiroudine, D. Beysens, Thermovibrational instability in supercritical fluids under weightlessness, Physical Review E 78 (3) (2008) 036325. 
[30] B. Zappoli, D. Beysens, Y. Garrabos, Heat Transfers and Related Effects in Supercritical Fluids, Springer, 2015.

[31] B. Shen, P. Zhang, An overview of heat transfer near the liquid-gas critical point under the influence of the piston effect: Phenomena and theory, International Journal of Thermal Sciences 71 (2013) 1-19.

[32] Nist standard reference database-refprop, version 8.0, 2006.

[33] Y. Masuda, T. Aizawa, M. Kanakubo, N. Saito, Y. Ikushima, One dimensional heat transfer on the thermal diffusion and piston effect of supercritical water, International Journal of Heat and Mass Transfer 45 (17) (2002) 36733677.

[34] Y. Mao, L. Guo, Numerical simulation for heat transfer mechanism of piston effect in supercritical water, Progress in Natural Science 16 (4) (2006) 457462(in Chinese).

[35] J. Straub, L. Eicher, A. Haupt, The process of heat and mass transport at the critical point of pure fluids, International journal of thermophysics 16 (5) (1995) 1051-1058.

[36] A. Haupt, J. Straub, Evaluation of the isochoric heat capacity measurements at the critical isochore of sf6 performed during the german spacelab mission d-2, Physical Review E 59 (2) (1999) 1795-1802.

[37] L. Wei, D. Yuan, B. Wu, D. Tang, A simulation for the piston effect in supercritical carbon dioxide with the non-flow model, Journal of Thermal Science 22 (5) (2013) 472-477. 
629

630

631

632

633

634

[38] N. Hasan, B. Farouk, Fast heating induced thermoacoustic waves in supercritical fluids: Experimental and numerical studies, Journal of Heat TransferTransactions of the Asme 135 (8).

[39] A. Onuki, R. A. Ferrell, Adiabatic heating effect near the gas-liquid critical point, Physica A: Statistical Mechanics and its Applications 164 (2) (1990) 254-264. 